\title{
相互作用对统计谱分析的影响
}

\section{- ${ }^{20} \mathrm{Ne}$ 原子核的形状因子}

\author{
赵恩广 李先㘫* 陈生忠 王游淮 \\ (中国科学院理论物理研究所, 北京)
}

我们曾经应用统计谱理论 ${ }^{[1]}$, 在平均场近似下, 讨论了部分 $s-d$ 壳原子核的形状因子 ${ }^{[n}$. 在不引入任何自由参数的条件下, 较好地再现了低动量区的实验结果; 对高动量区, 理论结果 在数量级和峰谷结构上也与实验定性符合，在某种意义下似乎表明，统计谱理论在描述原子 核某些基态性质上,也是很有希望的. 这一看法, 与不久前的研究结果一致 ${ }^{[3]}$. 同时, 它也启 示我们进一步去分析统计谱方法的固有优点. 实际上, 正是由于它既能不太困难地处理全部 有关核子的贡献, 又能尽可能多地考虑各种组态的贡献,使得统计谱理论更适于描述原子核的 整体性质，如讨论形状因子．同时，也使得它在分析相互作用性质时，能减少“等效”成分，使结 论更接近真实情况.

因此,本文仍以形状因子的统计谱分析为例，在 ${ }^{20} \mathrm{Ne}$ 原子核中研究相互作用对平均场近 似的修正,并探讨在这种分析中，验证相互作用性质的可能性.

按定义,原子核的形状因子可以写为

$$
F(q)=\frac{1}{z}\left\langle\boldsymbol{\phi}\left|\sum_{i=1}^{2} \exp \left(i q \cdot \boldsymbol{r}_{i}\right)\right| \boldsymbol{\phi}\right\rangle,
$$

在统计谱理论中,上式可写为

$$
F(q)-\frac{1}{z} \sum_{\nu}\left\langle\sum_{\alpha B} Q_{a B_{a}} a_{a}^{+} P_{\nu}(\hat{H})\right\rangle^{m} P_{\nu}(E),
$$

其中

$$
Q_{a \beta}-\langle\alpha|\exp (i \boldsymbol{q} \cdot \boldsymbol{r})| \boldsymbol{\beta}\rangle,
$$

$P_{v}(x)$ 是正交多项式,其表达式见文献[2]．上式中的 $\hat{A}$ 是原子核的哈密顿量, 这里写为

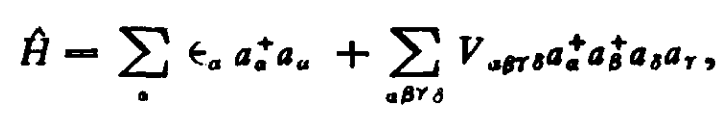

$\boldsymbol{\alpha}_{a}^{+}$是能量为 $\epsilon_{a}$ 的单粒子态 $|\alpha\rangle$ 的产生算符, $V_{\text {apro }}$ 是剩余相互作用的矩阵元, 其表达式为

$$
V_{a \beta \gamma \delta}-\frac{1}{2}[\langle\alpha \beta|V| \gamma \delta\rangle-\langle\alpha \beta|V| \delta \gamma\rangle],
$$

(2)式中其它符号的用法与文献 [2]相同.

因本文仅限于定性的分析, 我们把 $V$ 先取为一个简化的 8 -函数形式,

*现在工作单位: 北京大学物理系. 


$$
V-V_{0} \frac{\delta\left(r_{1}-r_{2}\right)}{r_{1} r_{2}} \delta\left(Q_{1}-Q_{2}\right),
$$

其中 $V_{0}$ 是力的强度. 在本文中, 它是唯一的可调参数,变化范围是 -30 到 $+30 \mathrm{MeVfm}$.

由文献 [2]的分析看出, $v=2$ 的多项式贡献不大. 这样, 在中心极限定理的作用下，(2) 式中对 $\nu$ 的求和, 可只取 0,1 两项. 对 $\alpha, \beta$ 等单粒子态的求和, 限制在 $1 s, 1 p, 1 d$ 和 $2 s$ 壳中 进行. 这相当于略去了 $2 p 1 f$ 以上壳层的贡献. 这在动量不大的情况下, 是可以的. 但是, 如 果形状因子中出现第三个极小值, 就应当考虑 $2 p 1 f$ 壳的影响. 需要指出的是, 对统计谱方法, 增加一两个壳层,并不会产生严重的计算困难.

把(3), (4),(5) 式代人 (2)式, 经过比较烦琐而直接的统计谱推导 ${ }^{[4]}$, 可以得到形状因子的 如下表达式:

其中

$$
\begin{aligned}
F(q)- & \frac{1}{Z d}\left[B_{1}\left(1+\left|C_{1}^{0}\right|^{2}+C_{1}^{0} C_{1}^{1} E\right)\right. \\
& \left.+\left(B_{2}+B_{3}\right)\left(C_{1}^{0} C_{1}^{1}+\left|C_{1}^{1}\right|^{2} E\right)\right]
\end{aligned}
$$

$$
\begin{aligned}
& B_{1}=\left(\begin{array}{c}
N-1 \\
Z-1
\end{array}\right) F_{3}, \\
& B_{2}-\left(\begin{array}{c}
N-2 \\
Z-2
\end{array}\right) F_{6}+\left(\begin{array}{c}
N-2 \\
Z-2
\end{array}\right) F_{5} E_{1}, \\
& B_{3}=4\left(\begin{array}{c}
N-3 \\
Z-2
\end{array}\right) V_{1}+2\left(\begin{array}{c}
N-3 \\
Z-3
\end{array}\right) F_{5} V_{2} \\
& C_{1}^{0}--M_{1} /\left(M_{2}-M_{1}^{2}\right)^{1 / 2} \text {, } \\
& C_{1}^{1}=1 /\left(M_{2}-M_{1}^{2}\right)^{1 / 2} \text {, } \\
& M_{1}=M_{1}^{0}+\frac{2}{d}\left(\begin{array}{c}
N-2 \\
Z-2
\end{array}\right) V_{2} \text {, } \\
& M_{2}=M_{2}^{0}+\frac{2}{d}\left\lceil 8\left(\begin{array}{c}
N-3 \\
Z-2
\end{array}\right) V_{3}+4\left(\begin{array}{c}
N-3 \\
Z-3
\end{array}\right) E_{1} V_{2}\right. \\
& +4\left(\begin{array}{c}
N-4 \\
Z-2
\end{array}\right) V_{4}+16\left(\begin{array}{c}
N-4 \\
Z-3
\end{array}\right) V \\
& \left.+4\left(\begin{array}{c}
N-4 \\
Z-4
\end{array}\right) V_{2}^{2}\right] \text {, }
\end{aligned}
$$

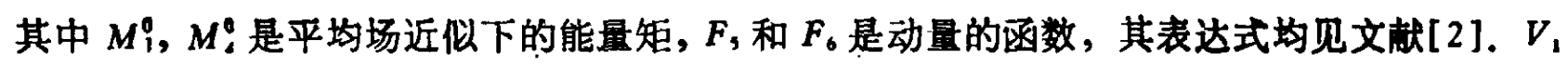
到 $V$, 的表达式如下:

$$
\begin{aligned}
& V_{1}-\sum_{a B V} Q_{a b} V_{B Y G T ;} \\
& V_{2}-\sum V_{a A A B}, \\
& V_{3}-\sum_{A B} \epsilon_{a} V_{A B A B}
\end{aligned}
$$




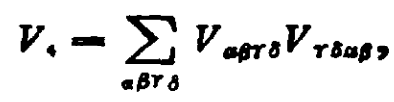

$$
\begin{aligned}
& V,-\sum_{a \beta Y \delta} V_{r \delta a \delta} V_{a g r B .}
\end{aligned}
$$

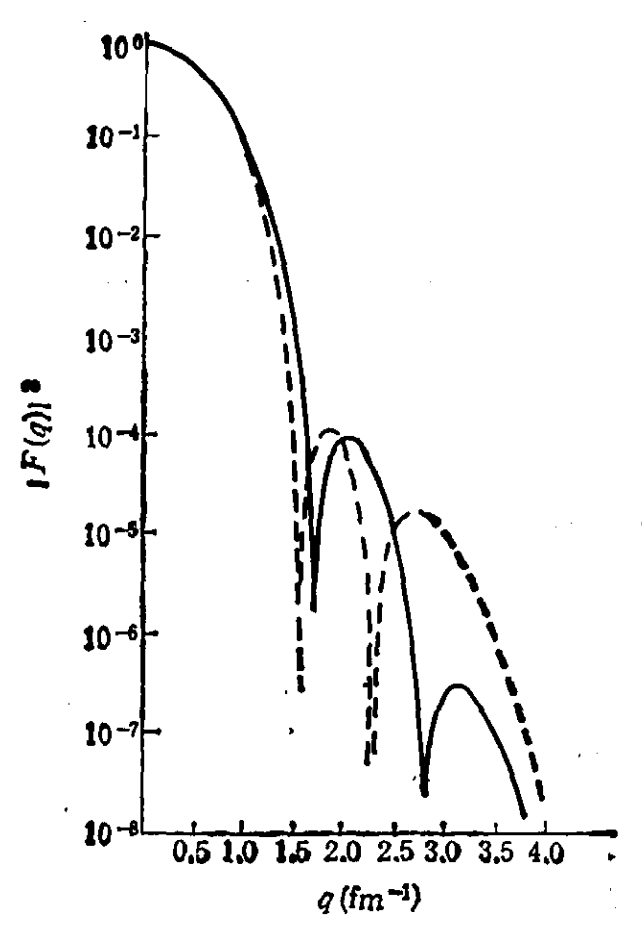

图 $1{ }^{20} \mathrm{Ne}$ 基态形状因子

一统计谱 $v_{0}=0$ 的结果;

- D统计橎 $V_{0}=-20 \mathrm{MeV}$ 的结果

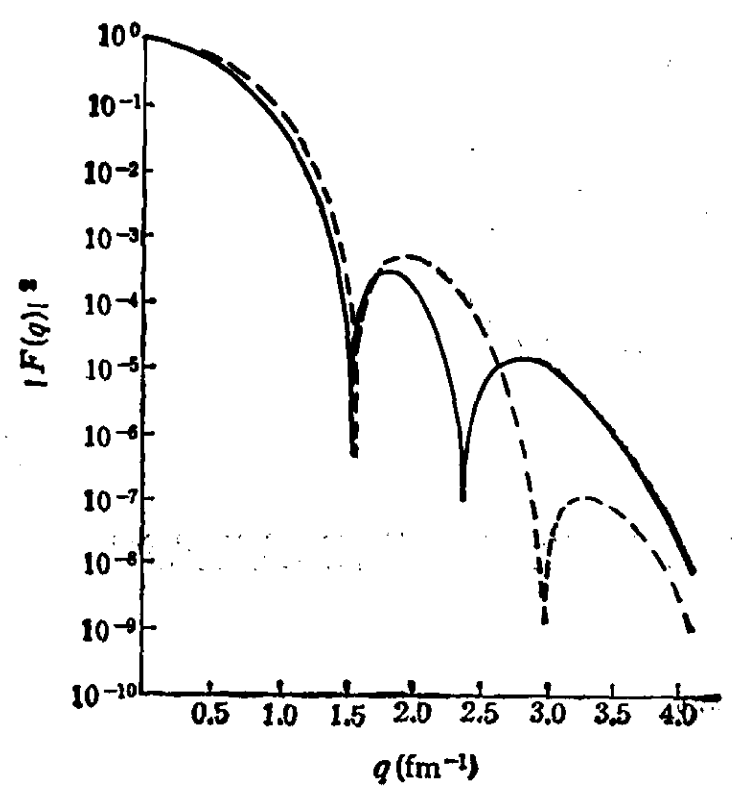

图 $3{ }^{20} \mathrm{Ne}$ 激发态形状因子 $E_{x}=50 \mathrm{MeV}$ 实线为 $V_{0}=0$ 的结果;

蛏线为 $V_{0}=+20 \mathrm{MeV}$ 结果

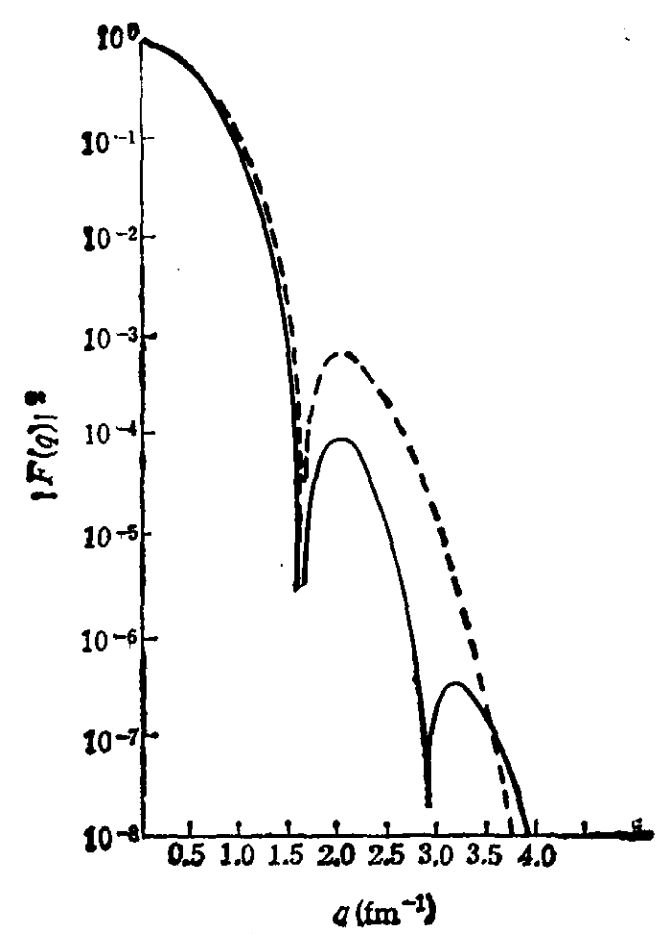

图 $2{ }^{80} \mathrm{Ne}$ 基态形状因子

$$
\text { 实线为 } V_{0}=0 \text { 结果; }
$$

䍀线为 $V_{0}=+20 \mathrm{MeV}$ 结果

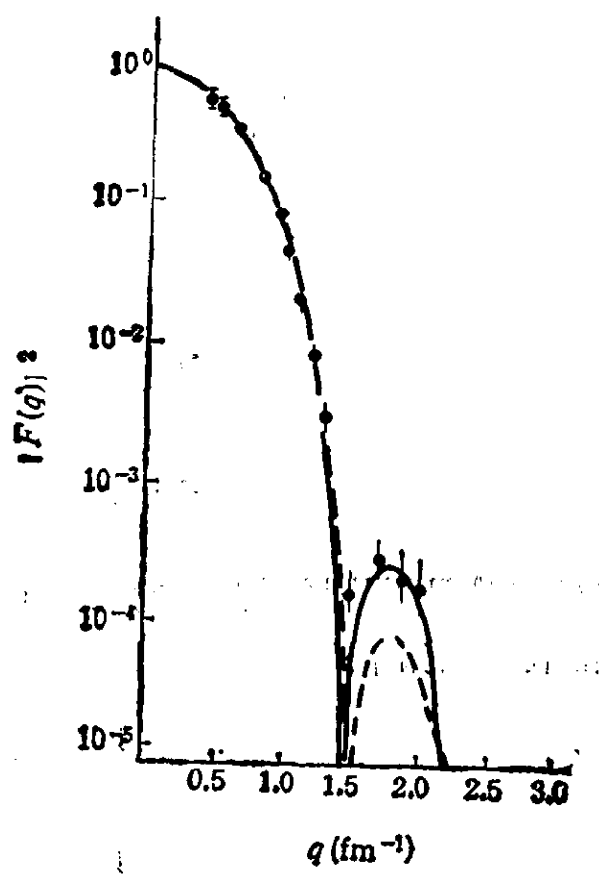

图 $4{ }^{20} \mathrm{Ne}$ 基态形状因子与实验比较 一统计谋结果; 
公式(6)是本文的基本公式. 利用它,在不同的核力强度 $V_{0}$ 下,计算了 ${ }^{20} \mathrm{Ne}$ 原子核基态和 激发态的形状因子. 下面给出一些典型结果, 分别画在图 1 到图 4 之中. 从这些结果的分析 中,我们可以看出:

第一，在统计谱计算中，相互作用的影响是重要的. 为了对比, 在前三个图中都给出了 $V_{0}=0$ 的计算结果. 很显然, 相互作用的 强度和符号, 对形状因子峰谷位置有重要影 响. 这就是说, 在统计谱计算中, 应当仔细地 处理相互作用; 同时,也可以通过这类工作研 究相互作用.

第二, 在我们的计算中, 当 $V_{0}$ 从零变到 $30 \mathrm{MeV}$ 时,排斥相互作用使形状因子的低动 量成分加大，高动量成分减少; 吸引相互作用 使低动量成分减少，高动量成分加大. 这有 助于人们了解相互作用对核子运动的影响. 在图 1 和图 2 中,给出了 $V_{0}- \pm 20 \mathrm{MeV}$ 的 计算结果.

第三, 在同一强度下, 激发态形状因子 所受核力影响较基态为小. 例如, 在基态时

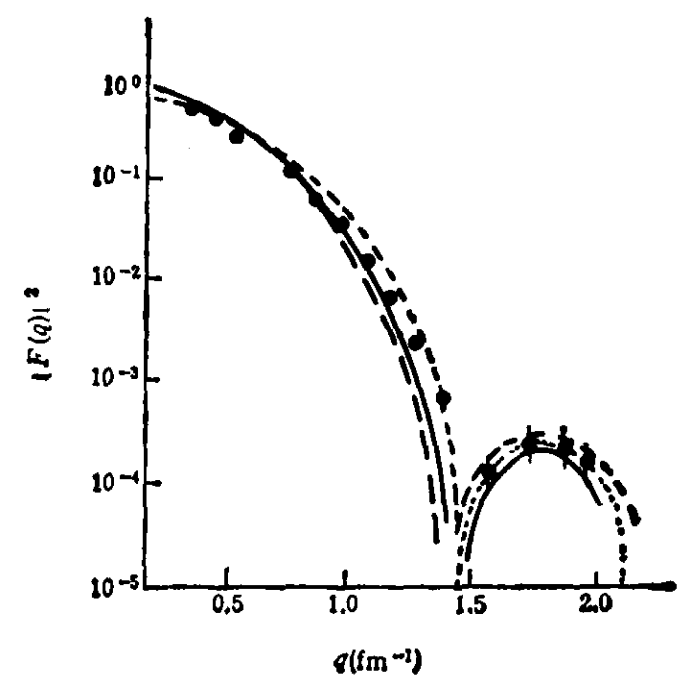

图 $5{ }^{20} \mathrm{Ne}$ 基态形状因子 斥力的形状因子当 $q$ 从 0 变到 $4 f^{-1}$ 时, 只有一个谷; 而 $V_{0}-0$ 的形状因子有两个谷. 当 $E_{x}-50 \mathrm{MeV}$ 时,在同样的动量范围内,二者就都有两个谷了.

为了和其它方法比较,在图 4 和图 5 中,我们还画出了采用 Brink-Boecker 位的辛群集体 模型的形状因子和采用 LINEG 等位的主壳混合 H-F 计算的形状因子 ${ }^{[6]}$. 从中可以看出, 与实验符合的程度,我们的结果比辛群的好,而与 H-F 计算相近. 但是, 从几种计算使用的 相互作用来看,显然统计谱的最简单. 这就暗示我们,由于统计谱方法能够尽可能多地考虑全 部有关的粒子和有关的组态,所以也就减少了相互作用中的等效贡献.

最后需要指出的是,若想更进一步比较不同方法和从中提取相互作用的知识,还应当采用 更现实的核力做统计谱计算, 同时也要对形状因子的高动量成分进行检验。遗情的是, 在 ${ }^{20} \mathrm{Ne}$ 情况下,目前还缺乏相应的实验数据。

\section{今 文嗝}

[1] Draayer, J. P., French, J. B., Wong, S. S. M., Ann. Phys., 106(1977), 472.

[2] 赵恩广、陈生忠, 原子核物理, 6 (1984), 42.

[ 3 ] Halemane, T. R., Abibas, A., J. Phys. Nucl. Phys, G7(1981), 1639.

[ 4 ] Chang, B. D. and Wong. S. S. M., Nucl. Phys., A294(1978), 19.

[5] Steshenko, A. Z., Nucl. Phys., A445(1985), 462.

[6] Rlpka, G., in Proc. of the Ins. Cont. on Electron Scatsering and Photoraction, Sendai, Japan, 1972, 7:

[7] Horikawa, Y. et al., Phys. Lett., $36 \mathbf{B}(1971), 9$. 